\title{
Simultaneous Approximation of a Set of Bounded Real Functions
}

\author{
By J. B. Diaz and H. W. McLaughlin
}

\begin{abstract}
The problem of simultaneous Chebyshev approximation of a set $F$ of uniformly bounded, real-valued functions on a compact interval $I$ by a set $P$ of continuous functions is equivalent to the problem of simultaneous approximation of two real-valued functions $F^{+}(x), F^{-}(x)$, with $F^{-}(x) \leqq F^{+}(x)$, for all $x$ in $I$, where $F^{-}$is lower semicontinuous and $F^{+}$is upper semicontinuous.
\end{abstract}

1. Formulation of the Approximation Problem. In this introductory section, which consists of nine "points," the "general problem of the simultaneous approximation of a family of functions" is formulated (see, in particular, point 4). Besides, a "heuristic derivation" of the basic equation (equation $\left(\mathrm{T}_{2}\right)$ of point 8 ) is given.

1. Let $g$ be a (finite) real-valued function defined for all real numbers $x$ on the finite-closed real number interval $[a, b]=\{x \mid a \leqq x \leqq b\}$, where $a<b$. The "norm," $\|g\|$, is defined to be

$$
\|g\|=\sup _{a \leqq x \leqq b}|g(x)| .
$$

If $g$ is not bounded in absolute value on $[a, b]$, then $\|g\|=+\infty$; otherwise, $\|g\|$ is a nonnegative number.

2 . Let $F$ be a nonempty set ("family") of real-valued functions $f$, defined on $[a, b]$. The set of functions $F$ is supposed to be uniformly bounded (in absolute value) on $[a, b]$; i.e., there is a nonnegative number $M$ such that $|f(x)| \leqq M$ for any function $f \in F$ and any number $x \in[a, b]$. Clearly,

$$
\|f\| \leqq M
$$

for any $f \in F$. (In the "general approximation problem" of point 4 below, the family $F$ is "the set of functions being approximated.")

3. Let $P$ be a nonempty set ("class") of real-valued, continuous functions $p$, defined on $[a, b]$. (In the "general approximation problem," the class $P$ is "the set of approximating functions"; usually, for $n$ a nonnegative integer, the class $P$ consists of all real polynomials of degree $\leqq n$.)

4. For the purposes of the present paper, the "general problem of the simultaneous approximation of the family $F$ by means of functions from the class $P$ " consists in the determination of the number

$$
\inf _{p \in P} \sup _{f \in F}\|f-p\| \text {. }
$$

(The formulation of this "general approximation problem," as given here, was suggested by the "problem of simultaneous approximation of two bounded functions

Received October 21, 1968.

This paper was presented to the American Mathematical Society at the August 1968 meeting at Madison, Wisconsin (see Abstract 658-200, Notices Amer. Math. Soc., v. 15, 1968, p. 778). 
$g_{1} \leqq g_{2}$, with $g_{1}$ lower semicontinuous and $g_{2}$ upper semicontinuous," of C. B. Dunham [1, p. 472]; this problem of Dunham will be discussed more fully under point 5 below.)

5. Consider, in particular, the very special case in which the family $F$ consists of a single function $f$, which is bounded in absolute value on $[a, b]$. In this case, the "general problem of the (simultaneous) approximation of the bounded function $f$ by means of functions from the class $P$ " consists in the determination of the number

$$
\inf _{p \in P}\|f-p\| \text {. }
$$

(Suppose, for the moment, that the function $f$ were allowed to be unbounded in absolute value, that is to say, $\|f\|=+\infty$. Then, for every continuous approximating function $p$ one would have that $\|f-p\|=+\infty$, and hence $\inf _{p} \in_{P}\|f-p\|=+\infty$ also. Therefore, the restriction that the function $f$ being approximated be bounded in absolute value is a natural requirement to make.)

Dunham [1, specially p. 476] showed that this "approximation problem of a single bounded function $f$ " is equivalent to the "simultaneous approximation problem of a certain pair of bounded functions $f^{-} \leqq f^{+}$, where $f^{-}$is lower semicontinuous, and $f^{+}$is upper semicontinuous, on $[a, b]$ ]" (the set of approximating functions $P$ used in [1] is "unisolvent of degree $n$ on $[a, b]$," and includes, as an important special case, the case when $P$ is the class of real polynomials of degree $\leqq n$ ). Specifically, this "equivalence" result of [1] can be formulated as follows: Let the function $f^{+}$be defined by

$$
f^{+}(x)=\inf _{\delta>0} \sup _{0 \leqq|x-y|<\delta} f(y),
$$

for $a \leqq x \leqq b$ (the function $f^{+}$, which is known to be upper semicontinuous, is sometimes called the "upper envelope" of the function $f$ on $[a, b])$. Similarly, let the function $f^{-}$be defined by

$$
f^{-}(x)=\sup _{\delta>0} \inf _{0 \leqq|x-y|<\delta} f(y),
$$

for $a \leqq x \leqq b$ (the function $f^{-}$, which is known to be lower-semicontinuous, is sometimes called the "lower envelope" of the function $f$ on $[a, b]$; and, clearly, $\left.f^{-}=-(-f)^{+}\right)$. Then the following equality holds:

$$
\inf _{p \in P}\|f-p\|=\inf _{p \in P} \sup \left\{\left\|f^{+}-p\right\|,\left\|f^{-}-p\right\|\right\},
$$

where, of course, one can replace "sup" by "max" on the right-hand side. It is to be noticed that, if the function $f$ being approximated is required, in addition, to be continuous on $[a, b]$ (as in the classical Chebyshev approximation problem), then both $f^{+}$and $f^{-}$coincide with $f$, and the two sides of the last equation are identical.

6. As was described in point 5, Dunham [1] was led to introduce the notion of simultaneous approximation in the particular case in which the family $F$ consists of a pair of functions, $f_{1}$ and $f_{2}$, with $f_{1} \leqq f_{2}$, where $f_{1}$ is lower-semicontinuous while $f_{2}$ is upper-semicontinuous. Further, Dunham, see [1, p. 477], also considered briefly the particular case when the family $F$ consists of a finite number of continuous functions, in which case it is stated that the problem of the simultaneous approximation of a finite number of continuous functions is equivalent to the problem of the 
simultaneous approximation of two continuous functions, namely, the minimum and the maximum of the finite set of continuous functions. It is natural to ask what, if anything, can be said when $F$ is an infinite set, say, countably infinite, to start out with. In [1], no mention is made of the simultaneous approximation problem when the set of functions $F$ is not finite.

7. It is the purpose of the present paper to show that a formula, analogous to that of point 5 , holds for any nonempty family $F$; this formula means, in words, that "the general problem of the simultaneous approximation of the family $F$ by means of functions from the class $P$ " is equivalent to the problem of the simultaneous approximation of a certain pair of functions $F^{-}$and $F^{+}$, with $F^{-} \leqq F^{+}$, where $F^{-}$is lower semicontinuous, and $F^{+}$is upper semicontinuous, on $[a, b]$ (that is, the simultaneous approximation problem for "any" family $F$ can be, at least theoretically, "reduced" to a simpler "Dunham type" approximation problem for a family consisting of only "two" functions $F^{-}$and $F^{+}$). Analytically, this result can be formulated as follows: Let the function $F^{+}$be defined by

$$
F^{+}(x)=\inf _{\delta>0} \sup _{0 \leqq|x-y|<\delta} \sup _{f \in F} f(y),
$$

for $a \leqq x \leqq b$; and let the function $F^{-}$be defined by

$$
F^{-}(x)=\sup _{\delta>0} \inf _{0 \leq|x-y|<\delta} \inf _{f \in F} f(y),
$$

for $a \leqq x \leqq b$. Then the following equality holds:

$$
\inf _{p \in P} \sup _{f \in F}\|f-p\|=\inf _{p \in P} \sup \left\{\left\|F^{+}-p\right\|,\left\|F^{-}-p\right\|\right\},
$$

where, of course, one can replace "sup" by "max" on the right-hand side. Clearly, one has that $F^{-} \leqq F^{+}$, from the definition of the functions involved. Also, the functions $\sup _{f} \in_{F} f$ and $\inf _{f} \in_{F} f$ are bounded in absolute value on $[a, b]$ (recall the constant $M$ of point 2). Moreover, since

$$
F^{+}=\left(\sup _{f \in F} f\right)^{+}
$$

(that is to say, see point 5 above, the function $F^{+}$is the "upper envelope" of the function $\sup _{f} \in_{F} f$ ), it follows that $F^{+}$is an upper semicontinuous function on $[a, b]$. Similarly, since

$$
F^{-}=\left(\inf _{f \in F} f\right)^{-},
$$

it follows that $F^{-}$is a lower semicontinuous function on $[a, b]$.

8. A heuristic "derivation" of the basic equation to be proved will now be given, in order to clarify the logical structure of the formal proof, which is given in detail in Section 2.

The equation to be proved (see point 7 above), written without "superfluous letters," is just

$\left(\mathrm{T}_{2}\right) \inf _{p \in P} \sup _{f \in F}\|f-p\|=\inf _{p \in P} \sup \left\{\left\|\left(\sup _{f \in F} f\right)^{+}-p\right\|,\left\|\left(\inf _{f \in F} f\right)^{-}-p\right\|\right\}$. 
Viewing equation $\left(\mathrm{T}_{2}\right)$ purely formally, as an equality between two products, and then applying the "cancellation law" (by simply "cancelling $\inf _{p} \in_{P}$ on both sides of the equation"), leads one to suspect that it may be true that, for any $p \in P$,

$$
\sup _{f \in F}\|f-p\|=\sup \left\{\left\|\left(\sup _{f \in F} f\right)^{+}-p\right\|,\left\|\left(\inf _{f \in F} f\right)^{-}-p\right\|\right\} .
$$

(After one has recovered from the initial shock of this "deduction" of (C) from $\left(\mathrm{T}_{2}\right)$, it is readily realized that all that is being said is that, if $(\mathrm{C})$ holds for any $p$ in $P$, then, upon taking the inf over $P$, equation $\left(\mathrm{T}_{2}\right)$ will follow.)

Returning to (C), since it presumably holds for any continuous function $p$ (because $P$ is just any set of continuous functions on $[a, b]$ ), it perhaps is valid when $p$ is just the identically zero function, which is a very special continuous function on $[a, b]$. That is, one is led to consider the equality

$$
\sup _{f \in F}\|f\|=\sup \left\{\left\|\left(\sup _{f \in F} f\right)^{+}\right\|,\left\|\left(\inf _{f \in F} f\right)^{-}\right\|\right\},
$$

and to conjecture that $\left(\mathrm{T}_{1}\right)$ implies $(\mathrm{C})$.

However, instead of $\left(T_{1}\right)$, it is rather easy to show directly that

$$
\sup _{f \in F}\|f\|=\sup \left\{\left\|\sup _{f \in F} f\right\|,\left\|\inf _{f \in F} f\right\|\right\} ;
$$

and hence, upon comparing the right-hand sides of equations $\left(\mathrm{L}_{1}\right)$ and $\left(\mathrm{T}_{1}\right)$, it is clear that the "missing link," in order to complete the chain of reasoning in a proof, "by retracing the steps," of the desired equation $\left(\mathrm{T}_{2}\right)$, is simply

$$
\sup \left\{\left\|\sup _{f \in F} f\right\|,\left\|\inf _{f \in F} f\right\|\right\}=\sup \left\{\left\|\left(\sup _{f \in F} f\right)^{+}\right\|,\left\|\left(\inf _{f \in F} f\right)^{-\|}\right\|\right\} .
$$

The structure of the formal proof of $\left(\mathrm{T}_{2}\right)$, given in Section 2, obtained by retracing the preceding heuristic steps, is then clear, and can be expressed as follows: $\left(\mathrm{L}_{1}\right)$ is the conclusion of Lemma $1 ;\left(\mathrm{L}_{2}\right)$ is the conclusion of Lemma $2 ;\left(\mathrm{T}_{1}\right)$ is the conclusion of Theorem 1 ; (C) is the conclusion of the Corollary; and, finally, $\left(\mathrm{T}_{2}\right)$ is the conclusion of Theorem 2 .

9. It is evident that, in this paper, the finite interval $[a, b]$ may be replaced throughout by a closed and bounded (i.e., compact) subset of the real numbers; and, in fact, even by a nonempty compact metric space, with only minor changes in the text.

2. Equivalence of the General Approximation Problem to a Simpler Approximation Problem. This section contains the formal proof of the basic equation $\left(\mathrm{T}_{2}\right)$, as outlined in point 8 of Section 1.

Lemma 1.

$$
\sup _{f \in F}\|f\|=\sup \left\{\left\|\sup _{f \in F^{*}} f\right\|,\left\|\inf _{f \in F} f\right\|\right\} .
$$

Proof. To save writing, denote

$$
u_{F}=\sup _{f \in F} f, \quad l_{F}=\inf _{f \in F} f ;
$$


then the equality to be proved is

$$
\sup _{f \in F}\|f\|=\max \left\{\left\|u_{F}\right\|,\left\|l_{F}\right\|\right\} .
$$

It will be shown first that

$$
\sup _{f \in F}\|f\| \leqq \max \left\{\left\|u_{F}\right\|,\left\|l_{F}\right\|\right\} .
$$

This inequality follows from the fact that, for all $f \in F$,

$$
l_{F}(x) \leqq f(x) \leqq u_{F}(x), \quad a \leqq x \leqq b .
$$

Therefore

which yields

$$
\begin{aligned}
|f(x)| & \leqq \max \left\{\left|u_{F}(x)\right|,\left|l_{F}(x)\right|\right\}, \\
& \leqq \max \left\{\left\|u_{F}\right\|,\left\|l_{F}\right\|\right\},
\end{aligned}
$$

$$
\|f\| \leqq \max \left\{\left\|u_{F}\right\|,\left\|l_{F}\right\|\right\}
$$

and hence (2) follows.

It remains to show that

$$
\sup _{f \in F}\|f\| \geqq \max \left\{\left\|u_{F}\right\|,\left\|l_{F}\right\|\right\} ;
$$

and, in the first part of this argument, it will be proved that

$$
\sup _{f \in F}\|f\| \geqq\left\|u_{F}\right\| \text {. }
$$

Let $\left\{x_{k}\right\}_{k=1}^{\infty}$ be a sequence of numbers, with $a \leqq x_{k} \leqq b$ for $k=1,2, \cdots$, such that

$$
\lim _{k \rightarrow \infty}\left|u_{F}\left(x_{k}\right)\right|=\left\|u_{F}\right\| .
$$

In the sequence of numbers $\left\{u_{F}\left(x_{k}\right)\right\}_{k=1}^{\infty}$, there are either infinitely many numbers $\geqq 0$, or else infinitely many $<0$; in the first case, it follows that there is an infinite subsequence $\left\{u_{F}\left(x_{n_{k}}\right)\right\}_{k=1}^{\infty}$, such that

$$
\lim _{k \rightarrow \infty} u_{F}\left(x_{n_{k}}\right)=\left\|u_{F}\right\|,
$$

while, in the second case, there is a subsequence $\left\{u_{F}\left(x_{n_{k}}\right)\right\}_{k=1}^{\infty}$, such that

$$
-\lim _{k \rightarrow \infty} u_{F}\left(x_{n_{k}}\right)=\left\|u_{F}\right\| \text {. }
$$

In either case, letting $\left\{x_{k}\right\}_{k=1}^{\infty}$ denote the chosen subsequence, if necessary, one has that

$$
\lim _{k \rightarrow \infty}\left|u_{F}\left(x_{k}\right)\right|=\left|\lim _{k \rightarrow \infty} u_{F}\left(x_{k}\right)\right|=\left\|u_{F}\right\| .
$$

Now, let $\left\{f_{k}\right\}_{k=1}^{\infty}$ be a sequence of functions from $F$ such that

$$
u_{F}\left(x_{k}\right)-1 / k \leqq f_{k}\left(x_{k}\right) \leqq u_{F}\left(x_{k}\right),
$$

for $k=1,2, \cdots$ (the existence of such a sequence of functions follows from the definition of $\left.u_{F}\right)$. Then, one has 


$$
\lim _{k \rightarrow \infty} f_{k}\left(x_{k}\right)=\lim _{k \rightarrow \infty} u_{F}\left(x_{k}\right)
$$

and

$$
\left|\lim _{k \rightarrow \infty} f_{k}\left(x_{k}\right)\right|=\left|\lim _{k \rightarrow \infty} u_{F}\left(x_{k}\right)\right|=\left\|u_{F}\right\|
$$

Hence, since

$$
\sup _{f \in F}\|f\| \geqq\left|\lim _{k \rightarrow \infty} f_{k}\left(x_{k}\right)\right|
$$

it follows that (4) holds.

Next, it will be proved that

$$
\sup _{f \in F}\|f\| \geqq\left\|l_{F}\right\| .
$$

Letting $\left\{\bar{x}_{k}\right\}_{k=1}^{\infty}$ be a sequence of numbers, with $a \leqq \bar{x}_{k} \leqq b$, for $k=1,2, \cdots$, such that

$$
\lim _{k \rightarrow \infty}\left|l_{F}\left(\bar{x}_{k}\right)\right|=\left|\lim _{k \rightarrow \infty} l_{F}\left(\bar{x}_{k}\right)\right|=\left\|l_{F}\right\|,
$$

and $\left\{\bar{f}_{k}\right\}_{k=1}^{\infty}$ be a sequence from $F$ such that

$$
l_{F}\left(\bar{x}_{k}\right) \leqq \bar{f}_{k}\left(\bar{x}_{k}\right) \leqq l_{F}\left(\bar{x}_{k}\right)+1 / k,
$$

for $k=1,2, \cdots$, one obtains (5). Alternatively, one may use, instead of the reasoning just given, the identity

$$
l_{F}=\inf _{f \in F} f=-\sup _{f \in F}(-f),
$$

plus the already proved inequality (4), in order to get (5).

Inequalities (4) and (5) give the desired equality, and the proof of Lemma 1 is complete.

Lemma 2. If $l$ and $u$ are bounded, real-valued functions defined on $[a, b]$, with $l \leqq u$, then

$$
\max \{\|u\|,\|l\|\}=\max \left\{\left\|u^{+}\right\|,\left\|l^{-}\right\|\right\} .
$$

Proof. It will be shown first that

$$
\max \{\|u\|,\|l\|\} \leqq \max \left\{\left\|u^{+}\right\|,\left\|l^{-}\right\|\right\} .
$$

This inequality follows from the fact that if $g$ is any real-valued function on $[a, b]$ such that

$$
\Gamma^{-}(x) \leqq g(x) \leqq u^{+}(x), \quad a \leqq x \leqq b,
$$

then

$$
\begin{aligned}
|g(x)| & \leqq \max \left\{\left|u^{+}(x)\right|,\left|l^{-}(x)\right|\right\} \\
& \leqq \max \left\{\left\|u^{+}\right\|,\left\|l^{-}\right\|\right\}
\end{aligned}
$$

and, consequently 


$$
\|g\| \leqq \max \left\{\left\|u^{+}\right\|,\left\|\tau^{-}\right\|\right\} .
$$

Since

$$
\tau^{-}(x) \leqq l(x) \leqq u^{+}(x), \quad a \leqq x \leqq b,
$$

and

$$
l^{-}(x) \leqq u(x) \leqq u^{+}(x), \quad a \leqq x \leqq b,
$$

it follows that (taking $g=l$ and $g=u$ in succession),

$$
\|l\| \leqq \max \left\{\left\|u^{+}\right\|,\left\|l^{-}\right\|\right\}
$$

and

$$
\|u\| \leqq \max \left\{\left\|u^{+}\right\|,\left\|l^{-}\right\|\right\},
$$

completing the proof of (6).

It remains to show that

$$
\max \{\|u\|,\|l\|\} \geqq \max \left\{\left\|u^{+}\right\|,\left\|l^{-}\right\|\right\} .
$$

This follows from the fact that, for any bounded real-valued function $g$ on $[a, b]$, it always happens that

$$
\|g\| \geqq\left\|g^{+}\right\|
$$

and

$$
\|g\| \geqq\left\|g^{-}\right\| .
$$

(The definitions of $g^{+}$and $g^{-}$are given under point 5 of Section 1.) Since $-(-g)^{+}=$ $g^{-}$, and $\|-g\|=\|g\|$, the second inequality will follow, once the first is established. To prove the first inequality, one needs the following formula:

$$
\|g\|=\sup _{a \leqq x \leqq b}|g(x)|=\max \left\{\sup _{a \leqq x \leqq b} g(x),-\inf _{a \leqq x \leqq b} g(x)\right\},
$$

which may be established thus:

For $x \in[a, b]$,

$$
|g(x)|=\max \{g(x),-g(x)\}
$$

therefore

$$
|g(x)| \leqq \max \left\{\sup _{a \leqq y \leqq b} g(y),-\inf _{a \leqq y \leqq b} g(y)\right\}
$$

and hence

$$
\|g\|=\sup _{a \leqq x \leqq b}|g(x)| \leqq \max \left\{\sup _{a \leqq y \leqq b} g(y),-\inf _{a \leqq y \leqq b} g(y)\right\} .
$$

On the other hand, for $x \in[a, b]$,

therefore

$$
|g(x)| \geqq g(x) ;
$$




$$
\sup _{a \leqq y \leqq b}|g(y)| \geqq g(x),
$$

and hence

$$
\sup _{a \leqq y \leqq b}|g(y)| \geqq \sup _{a \leqq x \leqq b} g(x) .
$$

Similarly,

$$
|g(x)| \geqq-g(x) ;
$$

therefore

$$
\sup _{a \leqq y \leqq b}|g(y)| \geqq-g(x)
$$

and hence

$$
\sup _{a \leqq y \leqq b}|g(y)| \geqq-\inf _{a \leqq x \leqq b} g(x) .
$$

Putting (11) and (12) together, one obtains

$$
\|g\|=\sup _{a \leqq x \leqq b}|g(x)| \geqq \max \left\{\sup _{a \leqq y \leqq b} g(y),-\inf _{a \leqq y \leqq b} g(y)\right\} ;
$$

and putting (10) and (13) together gives the formula $\left(^{*}\right)$.

Now to return to the proof of the inequality

$$
\|g\| \geqq\left\|g^{+}\right\| \text {. }
$$

Since, in view of formula $\left(^{*}\right)$,

$$
\left\|g^{+}\right\|=\sup _{a \leqq x \leqq b}\left|g^{+}(x)\right|=\max \left\{\sup _{a \leqq x \leqq b} g^{+}(x),-\inf _{a \leqq x \leqq b} g^{+}(x)\right\},
$$

the desired inequality will follow once it is proved that

$$
\sup _{a \leqq x \leqq b} g(x) \geqq \sup _{a \leqq x \leqq b} g^{+}(x),
$$

and

$$
\inf _{a \leqq x \leqq b} g(x) \leqq \inf _{a \leqq x \leqq b} g^{+}(x) .
$$

The proof of (14) is as follows: Since $g^{+}$is upper semicontinuous, there exists $\bar{x} \in[a, b]$ such that

$$
\sup _{a \leqq x \leqq b} g^{+}(x)=g^{+}(\bar{x}) .
$$

Now, by the definition of $g^{+}$, there exists a sequence $\left\{x_{k}\right\}_{k=1}^{\infty}$, with $a \leqq x_{k} \leqq b$ for $k=1,2, \cdots$, such that

$$
\lim _{k \rightarrow \infty} x_{k}=\bar{x},
$$

and also such that, for each positive integer $k$, one has

$$
g\left(x_{k}\right) \geqq g^{+}(\bar{x})-1 / k,
$$


from which (14) follows readily (actually, the equality sign holds in (14), since $g(x) \leqq g^{+}(x)$ for $x \in[a, b]$, and hence $\left.\sup _{a \leqq x \leqq b} g(x) \leqq \sup _{a \leqq x \leqq b} g^{+}(x)\right)$.

The proof of (15) is as follows: By the definition of $g^{+}$one has

$$
g(x) \leqq g^{+}(x), \quad a \leqq x \leqq b,
$$

and consequently (15) follows (that the equality sign does not always hold in (15) may be seen by choosing $g(x)$ to be unity for $a \leqq x<b$, and zero for $x=b$; the "theoretical reason" for this apparent discrepancy between (14) and (15) seems to be that, in the proof of (14), the fact that $g^{+}$attains the supremum of $g$ on $[a, b]$ is used, together with the inequality $g \leqq g^{+}$, while in the proof of $(15)$, only the inequality $g \leqq g^{+}$can be used, since $g^{+}$need not attain the infimum of $g$ on $[a, b]$ ).

It is clear that, putting $g=u$ in inequality (8), and $g=l$ in inequality (9), one obtains (7). Inequalities (6) and (7) give the desired equality, and the proof of Lemma 2 is complete.

THEOREM 1.

$$
\sup _{f \in F}\|f\|=\sup \left\{\left\|\left(\sup _{f \in F} f\right)^{+}\right\|,\left\|\left(\inf _{f \in F} f\right)^{-}\right\|\right\} .
$$

Proof. From Lemma 1 one has that

$$
\sup _{f \in F}\|f\|=\max \left\{\left\|\sup _{f \in F} f\right\|,\left\|\inf _{f \in F} f\right\|\right\} ;
$$

hence the desired conclusion follows from Lemma 2, upon choosing

$$
u=\sup _{f \in F} f, \quad l=\inf _{f \in F} f .
$$

Corollary. Let $p$ be a real-valued, continuous function on $[a, b]$. Then

$$
\sup _{f \in F}\|f-p\|=\sup \left\{\left\|\left(\sup _{f \in F} f\right)^{+}-p\right\|,\left\|\left(\inf _{f \in F} f\right)^{-}-p\right\|\right\} \text {. }
$$

Proof. As an auxiliary proposition, it will first be shown that, for any bounded real-valued function $h$ on $[a, b]$, one has

$$
[h-p]^{+}=h^{+}-p,
$$

and

$$
[h-p]^{-}=h^{-}-p .
$$

(Since, for any bounded real-valued function $f$ on $[a, b]$, one has

$$
-(-f)^{+}=f^{-}
$$

equation (17) is implied by equation (16), as is evidenced by the following simple computation:

$$
\begin{aligned}
(h-p)^{-}=-[-(h-p)]^{+}=-(-h+p)^{+} & =-\left[(-h)^{+}+p\right] \\
& =-(-h)^{+}-p \\
& \left.=h^{-}-p .\right)
\end{aligned}
$$

Now to prove (16). It will first be shown that 


$$
[h-p]^{+} \leqq h^{+}-p .
$$

To see this, let $x \in[a, b]$, and $\left\{x_{k}\right\}_{k=1}^{\infty}$ be a sequence of numbers from $[a, b]$ such that

$$
\lim _{k \rightarrow \infty} x_{k}=x,
$$

and

$$
\lim _{k \rightarrow \infty}\left[h\left(x_{k}\right)-p\left(x_{k}\right)\right]=[h-p]^{+}(x) .
$$

Then, since $p$ is continuous at $x$, one has

$$
\begin{aligned}
{[h-p]^{+}(x) } & =\lim _{k \rightarrow \infty}\left[h\left(x_{k}\right)-p\left(x_{k}\right)\right] \\
& =\lim _{k \rightarrow \infty} h\left(x_{k}\right)-\lim _{k \rightarrow \infty} p\left(x_{k}\right) \\
& =\lim _{k \rightarrow \infty} h\left(x_{k}\right)-p(x) \\
& \leqq\left[\lim _{\delta \rightarrow 0} \sup _{0 \leqq{ }^{\prime} x-y \mid<\delta ;} h \leqq y \leqq b\right. \\
& =h^{+}(x)-p(x),
\end{aligned}
$$

which gives (18).

It remains to show that

$$
[h-p]^{+} \geqq h^{+}-p .
$$

To see this, let $x \in[a, b]$, and $\left\{x_{k}\right\}_{k=1}^{\infty}$ be a sequence of numbers from $[a, b]$ such that

and

$$
\lim _{k \rightarrow \infty} x_{k}=x
$$

$$
\lim _{k \rightarrow \infty} h\left(x_{k}\right)=h^{+}(x) .
$$

Then, since $p$ is continuous at $x$,

which gives (19).

$$
\begin{aligned}
h^{+}(x)-p(x) & =\lim _{k \rightarrow \infty} h\left(x_{k}\right)-\lim _{k \rightarrow \infty} p\left(x_{k}\right) \\
& =\lim _{k \rightarrow \infty}\left[h\left(x_{k}\right)-p\left(x_{k}\right)\right] \\
& \leqq \lim _{\delta>0} \sup _{0 \leqq|x-y|<\delta ; a \leqq y \leqq b}[h(y)-p(y)] \\
& =[h-p]^{+}(x),
\end{aligned}
$$

Putting together inequalities (18) and (19) gives equation (16).

For convenience in writing, let $G=\{g \mid g=f-p$, where $f \in F\}$. The result of the corollary then follows from the previous theorem, applied to the family $G$, since, on the one hand

$$
\sup _{g \in G}\|g\|=\sup _{f \in F}\|f-p\| ;
$$

while, from (16), with $h=\sup _{f} \in F$, 


$$
\left(\sup _{g \in G} g\right)^{+}=\left[\left(\sup _{f \in F} f\right)-p\right]^{+}=\left(\sup _{f \in F} f\right)^{+}-p
$$

and, from (17)

$$
\left(\inf _{g \in G} g\right)^{-}=\left[\left(\inf _{f \in F} f\right)-p\right]^{-}=\left(\inf _{f \in F} f\right)^{-}-p .
$$

Now, let $P$ be a nonempty set of real-valued continuous functions on $[a, b]$. Taking the infimum over $P$, on both sides of the equality in the conclusion of the corollary, gives the following approximation theorem:

Theorem 2.

$$
\inf _{p \in P} \sup _{f \in F}\|f-p\|=\inf _{p \in P} \sup \left\{\left\|\left(\sup _{f \in F} f\right)^{+}-p\right\|,\left\|\left(\inf _{f \in F} f\right)^{-}-p\right\|\right\} .
$$

Remark. In words, this theorem states that the problem of simultaneous approximation of the family $F$, in the supremum norm, by functions of the class $P$, is equivalent to the simpler problem of approximating, simultaneously, two functions, a certain upper semicontinuous function and a certain lower semicontinuous function.

Rensselaer Polytechnic Institute

Troy, New York 12181

1. C. B. Dunham, "Simultaneous Chebyshev approximation of functions on an interval," Proc. Amer. Math. Soc., v. 18, 1967, pp. 472-477. MR $35 \# 3334$. 